\title{
Guía Metodológica para Definir el Perfil Profesional de Programas Académicos Mediante la Herramienta Ábaco de Régnier
}

\author{
Raúl J. Martelo(1), Natividad Villabona ${ }^{(2)}$ e Iris Jiménez-Pitre ${ }^{(3)}$ \\ (1) Universidad de Cartagena, Facultad de Ingeniería, Grupo de Investigación en tecnologías de las \\ Comunicaciones e Informática, GIMATICA, Avenida del Consulado, Calle 30, No 48 - 152, Cartagena - \\ Colombia. (e-mail: rmartelog1@unicartagena.edu.co) \\ (2) Universidad de Cartagena, Facultad de Ciencias Económicas, Avenida del Consulado, Calle 30, № 48 \\ - 152, Cartagena - Colombia. (e-mail: nvillabonag@unicartagena.edu.co) \\ (3) Universidad de la Guajira, Facultad de Ciencias Básicas y aplicadas, grupo de investigación BIEMARC, \\ Km 5 vía a Maicao, Riohacha - Colombia. (e-mail: iajimenez@uniguajira.edu.co)
}

Recibido May. 4, 2016; Aceptado Jun. 29, 2016; Versión final Sep. 20, 2016, Publicado Feb. 2017

\begin{abstract}
Resumen
El presente trabajo propone una guía metodológica que establece los pasos a seguir para el diseño de perfiles profesionales de programas académicos en instituciones de educación superior. Para ello, se utiliza la herramienta Ábaco de Régnier integrada a la plataforma web de prospectiva Softprosp, disponible en la literatura. La herramienta permite analizar el tema con grupos de expertos, para determinar las necesidades de formación que demanda el programa académico en estudio. Adicionalmente, esta guía facilita vincular expertos fuera de la zona geográfica donde se encuentra el objeto de estudio y eliminar sesgos. La propuesta estandariza parámetros para el diseño de perfiles profesionales en programas académicos y establece lineamientos suficientes para realizar procesos de gestión y control.
\end{abstract}

Palabras clave: perfil profesional, ábaco de Régnier, guía metodológica.

\section{Methodological Guide to Define the Professional Profile of Academic Programs by using the Regnier Abacus}

\begin{abstract}
This paper proposes a methodological guide to define the steps for the design of professional profiles of academic programs in higher education institutions. For this, the Regnier Abacus technique integrated with the prospective web platform Softprosp, available in the literature, was used. Such a technique allows analyzing the subject with groups of experts, to determine the formation needs that demand the academic program being studied. Additionally, this guide facilitates the inclusion of expert from other geographical area where the object of study is located, eliminating possible biases. The proposal standardizes parameters for designing professional profiles of academic programs and establishes sufficient guidelines to appropriately perform the processes of management and control.
\end{abstract}

Keywords: professional profile, Regnier's abacus, methodological guide 


\section{INTRODUCCIÓN}

En la Declaración Mundial sobre la Educación en el Siglo XXI, UNESCO (1998) insiste en que las instituciones de educación superior deben adecuar la formación profesional a necesidades actuales y futuras de la sociedad para hacer frente a nuevos retos. En el mismo sentido, la Política Educativa en Colombia se apoya en cuatro ejes estratégicos: cobertura, calidad, eficiencia y pertinencia; por lo que desde comienzos del milenio, instituciones de educación inician cambios en diseño y desarrollo de los planes de estudio para la formación de profesionales capaces de desempeñarse en cualquier contexto.

Tener presente la educación como eje de cambio y los factores que inciden en la elección de una institución de educación y de un programa académico según Huergo-Tobar (2014), destaca la confianza en las habilidades por adquirir como el factor más importante, que confirma la afirmación de Maringe (2006) sobre la existencia de un interés de utilidad en relación con la inversión en educación. El diseño de un programa académico es el resultado de decisiones que involucra a interesados de la comunidad educativa: estudiantes, egresados, empleadores, profesores, directivos, asociaciones gremiales (Tovar y Sarmiento, 2011); y cumple con la normatividad establecida por el Ministerio de Educación Nacional, máximo organismo rector de la educación en Colombia.

Las propuestas de diseños curriculares deben ser coherentes con el desarrollo económico, industrial y tecnológico en el objetivo de articular la educación superior y necesidades empresariales con una fuerza de trabajo que apoye su desarrollo e incremente su productividad. En consecuencia, cada programa académico delimita sus áreas de competencia y describe el quehacer académico para determinar contenidos y estrategias didácticas en el desarrollo de competencias profesionales y laborales (Sánchez et Al., 2011). En este proceso, el perfil profesional es la base del diseño curricular, que a su vez, determina el plan de estudios y sus contenidos; elementos en permanente actualización que se deben ajustar en el tiempo y tienen en consideración variables internas y externas que influyen en la formación académica (Escobar, 2013).

El perfil profesional determina las competencias del egresado para lograr el desempeño laboral según su área disciplinar; son las cualidades que demuestra para resolver problemas en un entorno de trabajo cambiante e incierto, y la capacidad para aprender y afrontar nuevas situaciones. La identificación de competencias con el proyecto Tuning inicialmente en Europa y luego en América Latina (2004-2007) ha sido un referente importante para universidades que inician procesos de modernización y actualización curricular. El referente para los programas de Administración, está dada por resultados de la investigación realizada con la coordinación de la Asociación Colombiana de Facultades de Administración -ASCOLFA- que bajo la metodología Tuning América Latina abordó la consulta sobre las competencias genéricas y específicas para analizar la importancia y obtención de cada grupo de competencias (ASCOLFA, 2010).

De cualquier forma, las instituciones de educación y los programas requieren información de diversas fuentes para identificar competencias en áreas de la profesión que atiendan la demanda a distintos contextos de desempeño, lo cual genera la necesidad de disponer de instrumentos o herramientas para agilizar el proceso de recolección, análisis y consolidación de resultados para cada perfil. Por lo anterior, se propone un enfoque mediante una guía metodológica para definir el perfil profesional de programas académicos haciendo uso de la herramienta Ábaco de Régnier. Para validar el enfoque propuesto, se aplicó la guía metodológica al programa de Administración de Empresas de la Universidad de Cartagena, con la finalidad de definir el perfil profesional. Además se encuentra que la aplicación de la guía presenta ventajas, que van desde el ahorro en costos de viáticos de expertos, mejoramiento en definición de variables a estudiar, gestión y control del proceso, entre otras, que permiten evidenciar la utilidad de la misma.

\section{OTROS ANTECEDENTES}

La información es la descripción de hechos y situaciones a partir de datos en un contexto definido, a partir de lo cual pueden tomarse decisiones (Tobón et Al., 2001). Según (Borko, 1968), denota la información como ciencia interdisciplinaria que investiga las propiedades y su comportamiento, las fuerzas que gobiernan el flujo y su utilidad, además de técnicas, manuales y mecánicas, del proceso informativo para su eficaz almacenamiento, recuperación y diseminación.

Dado que la información es considerada primordial en todo proceso, necesita ser organizada de manera sistemática y metódica, con una coherencia lógica y empírica, para alcanzar la mayor utilidad en el mejor orden posible, es decir, obtener y generar conocimiento (Vidal y Araña, 2012), por lo cual a mediados del siglo XX, explota el desarrollo de tecnologías capaces de procesar datos y generar información (Sadosky, 2000), al tiempo que profesiones y ciencias afines al tratamiento de la misma, como la estadística, economía, entre otras, colaboran con dicho proceso (Velázquez et al., 2014). 
En la década de los años 80 del siglo pasado, se cuenta con el surgimiento de una disciplina: la gestión de información, que replantea el mercado laboral del profesional de la información y demanda un nuevo tipo de profesional con importantes responsabilidades en el diseño y el desarrollo de sistemas informáticos en organizaciones. A su vez, la misma da paso a una disciplina paralela: la gestión del conocimiento, considerada como la teoría de gestión que responde a la adaptación de últimas innovaciones tecnológicas en el tratamiento de la misma (Vidal y Araña, 2012).

Los datos son la materia prima base de cualquier decisión, no generan juicios; la información es un mensaje que pretende cambiar la perspectiva de algo; el conocimiento es un conjunto de informaciones que permite aprender a realizar algo antes no sabido, o bien, para hacerlo mejor (Tobón et Al., 2001). Las instituciones de educación superior (IES), como entidades de gestión de información y conocimiento, necesitan herramientas para dicha gestión en los departamentos requeridos, para lo cual, el uso de sistemas de información (SI) se hace necesario (Araya, 2015). Sin embargo, el uso de SI no garantiza una completa y adecuada gestión de la información y del conocimiento, por lo que se crean planes y guías metodológicas para tales fines, como ejemplo para gestión de la información en (Martelo et al., 2016), se proporciona una guía en la que se consolidan los pasos a seguir en el diseño de planes estratégicos informáticos en IES.

Para la gestión del conocimiento en IES, se puede analizar la creación de perfiles profesionales, los cuales se definen de múltiples formas, entre esas la mencionada en Morales et. Al. (2014), donde se determina el perfil desde el enfoque por competencias, realizando consultas por medio de encuestas y talleres a profesores de secundaria en servicio, académicos en ejercicio, entre otros actores. Por igual, se destaca el ejemplo de un estudio prospectivo aplicado al análisis e influencia de escenarios posibles sobre los perfiles de oficiales, suboficiales y tropa española en 2015 (Alba, 2010). En este escrito se hace uso de un estudio prospectivo de impactos cruzados, el cual aproxima a diferentes contextos potenciales en el horizonte temporal del 2015, teniendo en cuenta varias hipótesis de futuro.

En situaciones de cambios acelerados, diferentes tendencias e incremento de incertidumbre, la prospectiva es una herramienta fundamental para observar el futuro mediante espacios de debate y conversación mediada por expertos que examinan variables que puedan afectar la búsqueda de ese futuro deseado (Cassingena, 2003). Los primeros trabajos sobre prospectiva de Godet (2007) buscan el desarrollo de una metodología sistemática para la identificación de escenarios y definición de las líneas de acción y toma de decisiones anticipada para disminuir riesgos en el diseño y construcción del futuro. La opinión cualitativa de los expertos se convierte en cuantitativa mediante las herramientas de trabajo propuestas por Godet en su libro Prospectiva Estratégica: problemas y métodos (2007): Análisis MIC MAC, análisis MACTOR, análisis de escenarios, Ábaco de Régnier y árboles de pertinencia. La tabla 1 resume cuál es el propósito de cada una de ellas:

Tabla 1. Herramientas de Prospectiva (Godet, 2007)

\begin{tabular}{|l|l|}
\hline $\begin{array}{l}\text { Análisis MIC MAC O } \\
\text { Análisis estructural }\end{array}$ & $\begin{array}{l}\text { Describe los sistemas mediante una matriz con el propósito de identificar } \\
\text { las variables claves y la influencia entre las mismas. }\end{array}$ \\
\hline Análisis MACTOR & $\begin{array}{l}\text { Tiene como objetivo la valoración de las relaciones de fuerza entre los } \\
\text { actores de un sistema para comprender sus convergencias y } \\
\text { divergencias respecto a cierto número de posturas y de objetivos } \\
\text { asociados. }\end{array}$ \\
\hline Análisis de escenarios & $\begin{array}{l}\text { Su objetivo es estudiar en tiempo actual una situación bajo la influencia } \\
\text { de diferentes variables para presentarla a futuro en forma de diferentes } \\
\text { escenarios }\end{array}$ \\
\hline Ábaco de Régnier & $\begin{array}{l}\text { Permite la consulta de expertos, dando la oportunidad a todos de } \\
\text { expresar su opinión. No busca el consenso frente a un tema sino } \\
\text { conocer la opinión de todo el grupo participante. }\end{array}$ \\
\hline Árboles de pertinencia & $\begin{array}{l}\text { Presenta información como una estructura jerárquica, a partir de un nivel } \\
\text { general a niveles de mayor detalle. }\end{array}$ \\
\hline
\end{tabular}

La prospectiva ha tenido múltiples aplicaciones en diversos campos desde la determinación de políticas gubernamentales para el desarrollo nacional o local, los estudios sectoriales sobre las tendencias en educación, salud o energía, desarrollo tecnológico, investigación de mercados y el comercio internacional. Variados son los trabajos de investigación que muestran su aplicación en organizaciones públicas y privadas de diferentes sectores a mencionar el proceso de diseño y construcción del futuro empresarial (Quiroga, 2008), la determinación del perfil gerencial para empresas prestadoras de salud (Pacheco, 2008), el impacto de avances tecnológicos en el desarrollo económico de naciones (Bañuls y Salmerón, 2008), retos de ingeniería para desarrollo tecnológico de agroindustria (Castellanos et al., 2011), el estudio prospectivo de empresas de industria alimentaria (Hernández et al., 2012), entre otros. En el sector de la educación se pueden señalar trabajos como el diseño del perfil profesional que caracteriza al egresado de 
Licenciatura en Ciencias Farmacéuticas (Ortega et al., 2011), la integración dentro de las políticas y estrategias tecnológicas de la prospectiva para la gestión estratégica del conocimiento y su papel en grupos de investigación de universidades (Pineda, 2013).

El uso de la herramienta Ábaco de Régnier ofrece alternativas para comparar el punto de vista de un grupo con otros, a la vez, toma conciencia de la variedad de opiniones frente a un tema específico (Mojica, 1991) y así, evitar la creación de sesgos que líderes o expertos puedan producir al querer imponer ideas (Correa, 2010). Se citan algunos estudios que aplican la herramienta como prioridades de investigación para el control del cáncer en Colombia (Arias et al., 2008), agenda prospectiva estratégica de ciencia y tecnología (Sierra et al., 2013) y metodología para el diseño de perfiles basados en el enfoque de competencias (Canquiz y Inciarte, 2009), entre las aplicaciones que tiene para diversos sectores.

Cabe resaltar que la herramienta Ábaco de Régnier cuenta con características como las siguientes: Permite efectuar un análisis rápido y didáctico en el tratamiento de un problema, facilita la Intercomunicación, el debate y el intercambio de ideas más que llegar a un consenso; Permite a los que divergen expresarse (y valorar sus opiniones); Se anula casi en la totalidad la presencia de un líder (quien puede influenciar en el grupo). También es importante tener en cuenta que presenta algunas desventajas como: La herramienta no es muy conocida y resta tiempo al tener que dar una explicación sobre lo que significa para entender su fin (lo cual es facilitado con la herramienta SoftProsp); la herramienta modifica el funcionamiento habitual de un grupo, de ahí la dificultad de convencer a los que deben decidir para su utilización: el jefe puede encontrarse aislado, ya que el método obliga a todos los miembros del grupo a dar su opinión, a "anunciar su color". Lo anterior debido a la pluralidad de las opiniones permite ser enriquecedor, dado que no se ve marcada la influencia del jefe y por ende los sesgos.

Con herramientas como la anterior, al ser implementadas y aplicadas en empresas o instituciones a través de TIC's, hacen considerar a estas últimas factor clave, debido a que con ellas se goza de excelencia operacional y prestación de servicios efectivos, eficientes y oportunos (Griffin-Sobel, 2009), hecho que beneficia a la aplicación de la herramienta Ábaco de Régnier, al superar límites geográficos e integrar grupos para valorar temas y obtener resultados. En conclusión, con las ventajas de la herramienta Ábaco de Régnier, el presente estudio hace referencia al sector de educación superior para la definición del perfil profesional del Programa de Administración de Empresas de la Universidad de Cartagena.

\section{ESQUEMA PLANTEADO}

La metodología para el diseño de perfiles profesionales en programas académicos es participativa, de permanente reflexión y de consensos en los pasos que se requieren, siguiendo lineamientos del Ministerio de Educación Nacional de Colombia, según se muestra en la Fig. 1. La figura anterior representa las fases de la metodología para el diseño curricular. Al vincular la herramienta Ábaco de Régnier al proceso descrito, forman un nuevo esquema el cual se observa en la Figura 2.

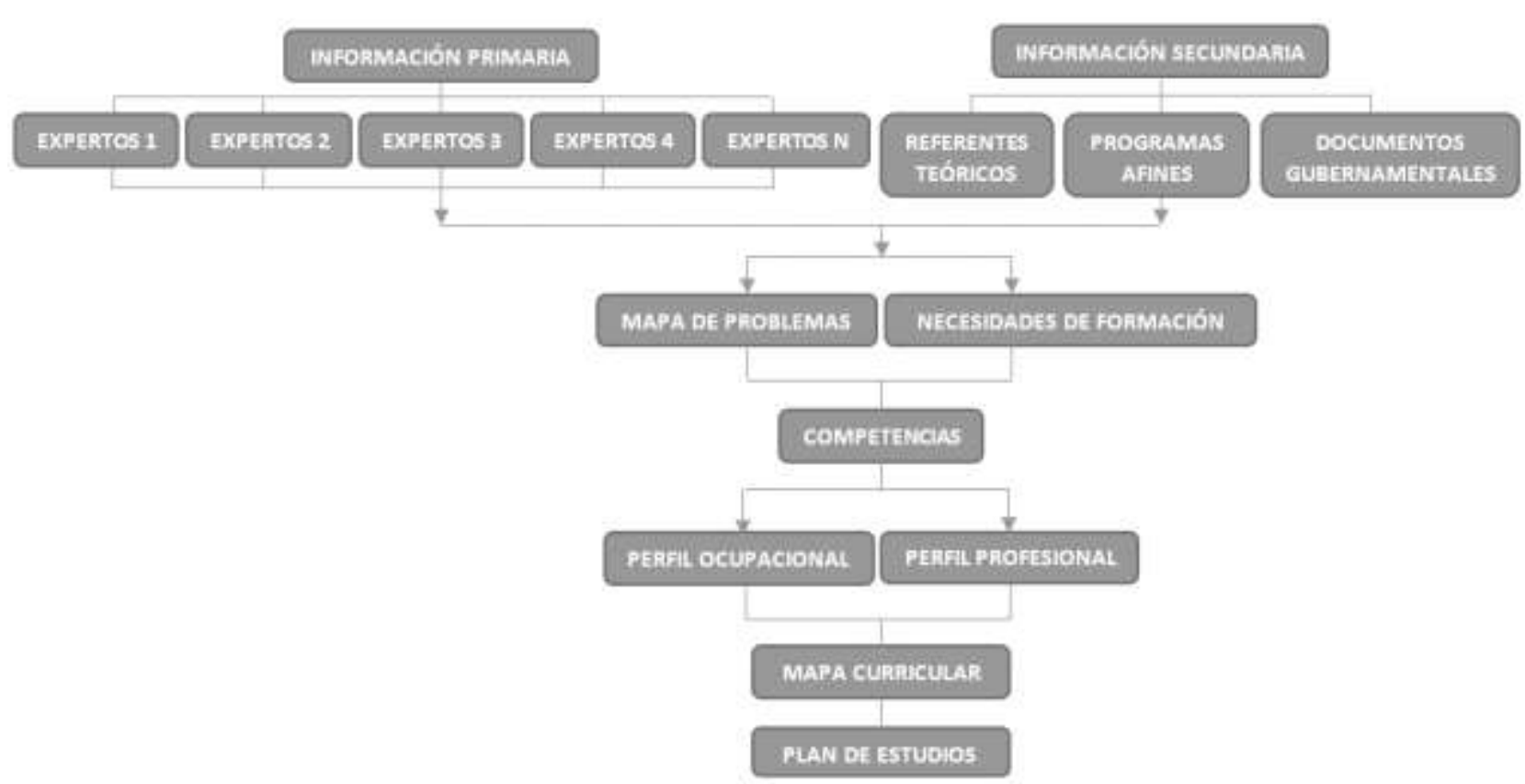

Fig. 1: Diagrama de metodología de diseño curricular. 


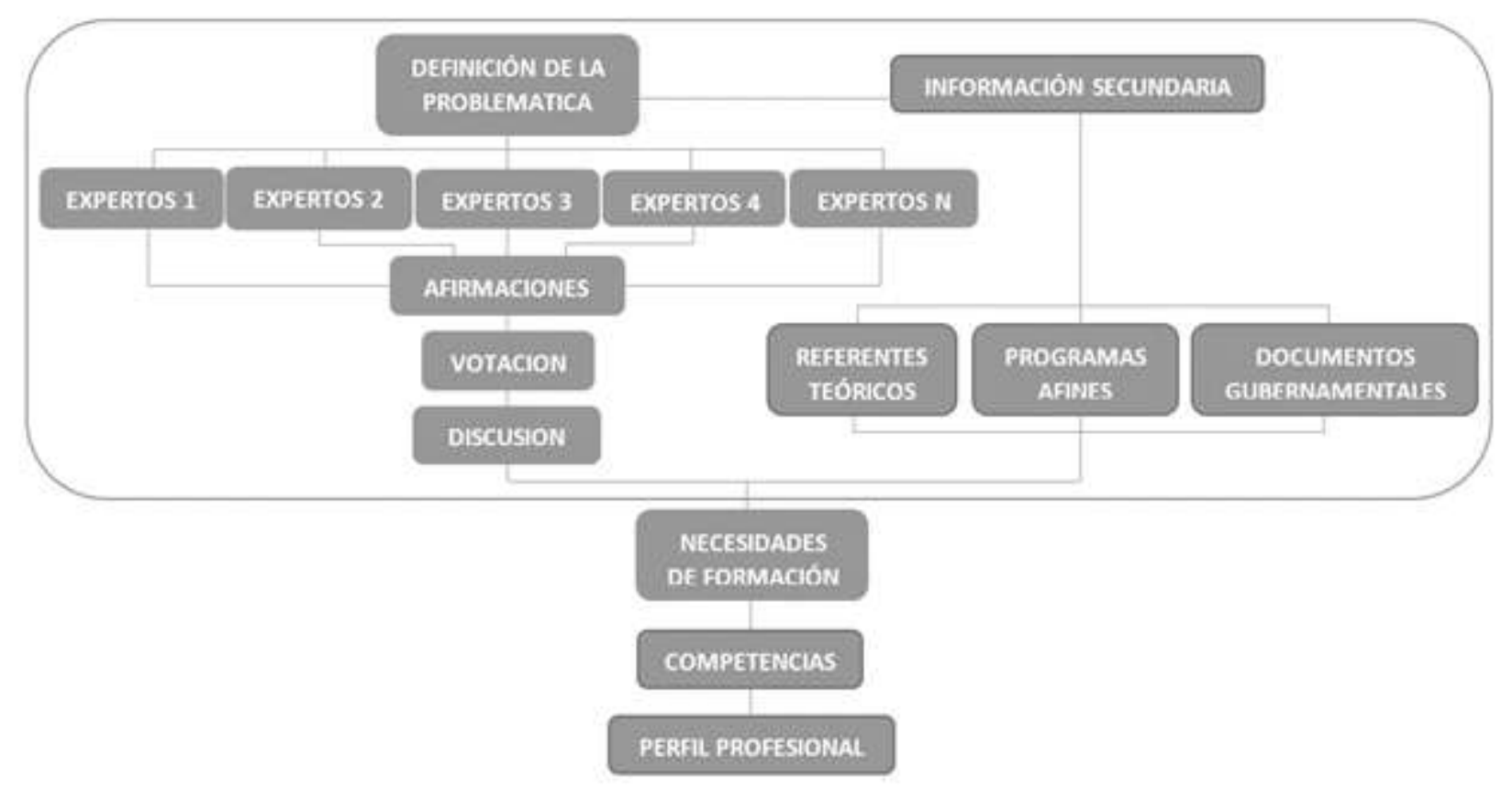

Fig. 2: Diagrama de metodología de diseño curricular vinculando Ábaco de Régnier.

EL proceso para definir el perfil profesional de un programa, es la base fundamental para el diseño curricular por competencias, Dicho esquema es descrito de la siguiente manera:

i) Definición de la problemática: Se requiere la recolección de información de fuentes primarias y secundarias para identificar competencias del egresado de un programa académico. La recopilación de fuentes primarias se realiza mediante entrevistas de profundidad y/o cuestionarios estructurados a estudiantes, egresados, docentes y empleadores. De igual manera, la información de fuentes secundarias se obtiene a partir de referentes teóricos, experiencias de programas afines y estudios e investigaciones de asociaciones propias de la profesión, documentos sobre tendencias y prospectivas de sectores económicos y políticas gubernamentales nacionales y regionales.

ii) Conformación de grupos de expertos: Las partes interesadas o stakeholders (estudiantes, egresados, docentes y empresarios), quienes evalúan el impacto de un programa académico; así como otras fuentes de interés que suministran información en el área del programa, como el sector gubernamental, asociaciones gremiales y profesionales conforman los grupos de expertos reflejados en la Fig. 2 como: expertos 1, expertos 2, expertos 3 , expertos 4 y expertos $\mathrm{N}$ (son posibles $\mathrm{N}$ cantidad de expertos para la definición del perfil profesional).

iii) Afirmaciones: De las entrevistas de profundidad se obtiene información sobre: actividades propias del profesional en su desempeño, necesidades de formación y problemas profesionales. De los cuestionarios estructurados, se consigue información sobre tendencias de los programas de formación y tipo de profesional requerido en las empresas; de los empleadores, adicionalmente se obtiene información sobre los requerimientos en los distintos desempeños en ejercicio de su profesión en las diferentes áreas.

iv) Votación: Cada experto vota en un formulario compuesto por las afirmaciones obtenidas anteriormente, proporcionando la calificación y el argumento o justificación de esta. Las afirmaciones, expertos y sus respectivos votos son organizados en una matriz de resultados, la cual podrá ser consultada por los participantes en el proceso de votación. Paralelo a las votaciones, los participantes tendrán la posibilidad de observar resultados, argumentos de cada respuesta y, en caso tal, cambiar el voto.

v) Discusión: Realizadas las votaciones se evalúan de manera interna: ítems y expertos participantes, para dar un veredicto sobre ítems más acertados, con la finalidad de crear: actividades, necesidades, tendencias, entre otra, referentes al programa académico en estudio.

vi) Análisis y determinación de necesidades de formación: De las entrevistas de profundidad se obtiene información sobre: actividades propias del egresado en su desempeño, necesidades de formación y problemas profesionales. De los cuestionarios estructurados, se consigue información sobre tendencias de 
los programas de formación y tipo de profesional requerido en las empresas; de los empleadores, adicionalmente se obtiene información sobre los requerimientos en los distintos desempeños en ejercicio de su profesión en las diferentes áreas.

vii) Establecer competencias. Los resultados de la fase anterior llevan a determinar, en reuniones con docentes del programa académico, las competencias que el estudiante debe desarrollar en el proceso de formación; por lo general lo deciden en comités curriculares del programa en estudio.

viii) Definir perfil profesional: A partir de la definición de competencias, y necesidades del entorno empresarial se establece el perfil profesional del futuro egresado.

\section{RESULTADOS}

Como caso de estudio se realizó la definición del perfil profesional del administrador del Programa de Administración de Empresas de la Universidad de Cartagena, creando una sesión de estudio con la herramienta Ábaco de Régnier en la plataforma SoftProsp, en donde se ingresan datos como nombre del caso a estudiar, fecha de inicio y fin, expertos, coordinadores. El estudio fue realizado en el segundo semestre del año 2015 en un periodo de dos meses. De acuerdo al esquema planteado en la figura 2, las fases se efectuaron de la siguiente manera:

En la fase de recolección de información se identifican y contactan los grupos de interés y se obtiene información secundaria a partir de documentos sobre las tendencias y prospectivas de los sectores económicos, políticas gubernamentales nacionales y regionales, experiencias de programas afines y de estudios e investigaciones de las asociaciones propias de la profesión (ASCOLFA, Consejo Profesional de Administración) y el documento "Proyecto Tuning Latinoamérica" que ofrece puntos de referencia y convergencia para que las instituciones de educación dispongan de sistemas educativos que faciliten la convalidación de titulaciones y movilidad estudiantil a nivel nacional e internacional. Los roles identificados que conforman los grupos de expertos fueron los mostrados en la Tabla 2.

Tabla 2. Roles identificados 2015.

\begin{tabular}{|l|l|}
\hline Expertos & Descripción \\
\hline Estudiantes & $\begin{array}{l}\text { Representantes estudiantiles del programa en diferentes } \\
\text { semestres }\end{array}$ \\
\hline Docentes & Docentes de las áreas del programa \\
\hline Egresados & $\begin{array}{l}\text { Docentes y profesionales vinculados a los sectores } \\
\text { productivos de la ciudad o región }\end{array}$ \\
\hline Empresarios & $\begin{array}{l}\text { Directivos de empresas que tienen en sus } \\
\text { organizaciones como empleados a estudiantes en } \\
\text { práctica o egresados del programa }\end{array}$ \\
\hline Gremios gubernamentales & $\begin{array}{l}\text { directivos vinculados a empresas del sector público o a } \\
\text { gremios área }\end{array}$ \\
\hline Asociaciones & $\begin{array}{l}\text { Directivos vinculados a asociaciones del a } \\
\text { profesional }\end{array}$ \\
\hline
\end{tabular}

De cada rol se toma una muestra representativa (Tabla 3), la cual conformará el grupo de expertos del estudio en mención. Cada participante de la muestra proporciona información acerca de: ¿Carencias de desempeño a los administradores de empresas en las diferentes áreas de desempeño?, ¿Cuáles son las áreas específicas de estudio que usted considera en que se debe formar un administrador de empresas?, ¿Cuáles son los problemas que habitualmente presentan los administradores de empresa en su desempeño en las áreas funcionales de las empresas? y ¿Qué sugerencia tiene frente al proceso de formación de los administradores de empresas de la Universidad de Cartagena?. Cada integrante de la muestra es agregado en el campo de expertos del formulario para creación de sesiones de estudio con la herramienta ábaco de SoftProsp.

Con la recolección de distintos tipos de información sobre el contexto y evolución de la profesión del administrador de empresas; estado de formación y cambios esperados; tendencias y proyecciones del futuro en relación con el contexto de desempeño; se da por hecha la fase afirmaciones, culminándola con el agregado de dicha información a la sección de creación de ideas de SoftProsp (Fig. 3). 
Tabla 3. Población y muestra de grupo de expertos 2015.

\begin{tabular}{|l|l|l|}
\hline Expertos & Población & Muestra \\
\hline Estudiantes & 866 & 267 \\
\hline Docentes & 61 & 53 \\
\hline Egresados & 657 & 243 \\
\hline Empleadores & 5 & 5 \\
\hline Gremios gubernamentales & 1 & 1 \\
\hline Asociaciones & 2 & 2 \\
\hline
\end{tabular}

\section{* Inicio / Crear items en ábaco de regnier \\ Crear ideas en Ábaco de Regnier}

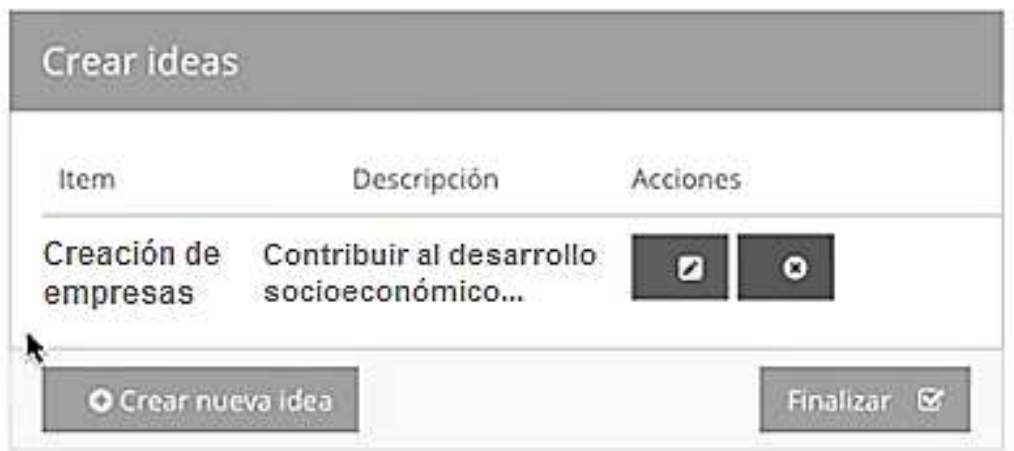

Fig. 3. Creación de ideas en Ábaco de Régnier

Con la anterior etapa terminada, se da paso a la fase de votación (Fig. 4), tomando ventaja de las bondades de conectividad de la plataforma, permitiendo realizarlas desde cualquier punto geográfico con acceso a internet y con total anonimato, eliminando sesgos por niveles jerárquicos, entre otros. Las votaciones en la plataforma se realizan de igual manera que en el mundo real, utilizando la escala de colores respectiva junto a un comentario el cual representa la justificación del voto efectuado.

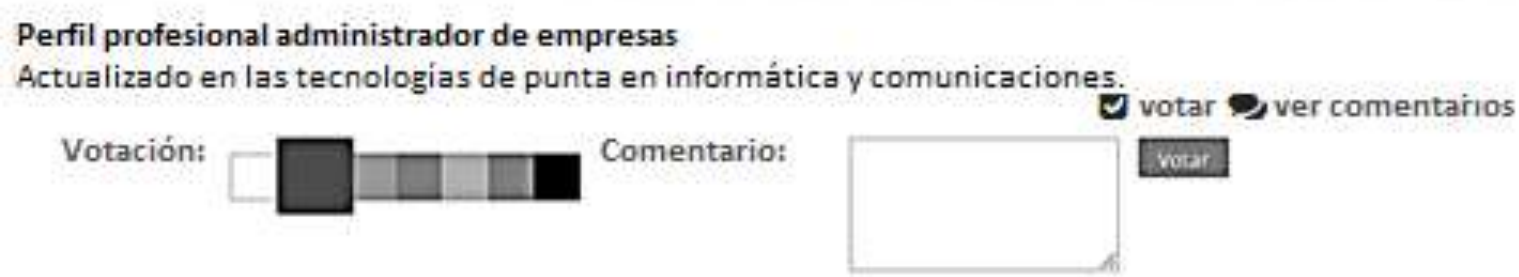

Fig. 4. Panel de votación Ábaco de Régnier

Finalizadas las votaciones, se procede a ejecutar la fase de discusión, llegando a la consolidación de resultados para la identificación de problemas y necesidades de formación. Lo anterior se alcanza priorizando ítems, ideas o afirmaciones votadas en la anterior fase por medio de sus puntuaciones, siendo de mayor impacto aquellas con puntuación más alta, es decir, con mayor favorabilidad entre el grupo de expertos. Las apreciaciones se encuentran disponibles en la sección ver resultados de la sesión ábaco en estudio, dentro de la plataforma SoftProsp. A partir de estos resultados se determinaron los siguientes puntos:

(i) Problemas profesionales: Falta liderazgo y definición estratégica, baja capacidad de análisis y manejo de sistemas, falta proactividad para tomar decisiones, baja habilidad de idioma inglés, deficiencia en la interpretación de textos, problemas de comunicación oral y deficiencia para identificar problemáticas.

(ii) Necesidades de formación: Están en relación con los problemas y tienen que ver con manejo de tecnologías informáticas y de comunicación, desarrollo de habilidades investigativas, cursos de crecimiento personal, comprensión lectora y toma de decisiones. 
(iii) Se determinan las competencias que el administrador de empresas debe desarrollar en el proceso de formación: Diseñar e implementar un planeamiento estratégico, táctico y operativo; administrar un sistema logístico integral; desarrollar, implementar y gestionar sistemas de control administrativo; elaborar, evaluar y administrar proyectos empresariales en diferentes tipos de organizaciones; interpretar la información contable y la información financiera para la toma de decisiones gerenciales; usar la información de costos para el planeamiento, el control y la toma de decisiones; tomar decisiones de inversión, financiamiento y gestión de recursos financieros en la empresa; ejercer el liderazgo para el logro y consecución de metas en la organización; administrar y desarrollar el talento humano en la organización; mejorar e innovar los procesos administrativos; detectar oportunidades para emprender nuevos negocios y/o desarrollar nuevos productos; utilizar las tecnologías de información y comunicación en la gestión; formular planes de marketing. A partir de la definición de competencias, y necesidades del entorno empresarial globalizado, el programa asume necesaria la formación de un administrador de empresas holístico que responda al perfil profesional.

(iv) Perfil Profesional: Gestionar los recursos necesarios para el logro de los objetivos de la organización; dirigir y coordinar la conformación del equipo humano pertinente con el tipo de organización; diseñar e implementar metodologías y herramientas de control y evaluación de las actividades administrativas; comprender la realidad humana y social del medio en que desarrolla su actividad profesional, para actuar con ética y responsabilidad social; diseñar, asimilar e implementar políticas, estrategias y sistemas en beneficio del logro y mantenimiento de los niveles de productividad; contribuir al desarrollo socioeconómico mediante la creación de empresa.

(v) Contextos de desempeño: Empresas industriales, comerciales o de servicios; dirección o división del área de talento humano; jefatura o coordinación de programas de desarrollo y entrenamiento de personal, dirección administrativa y/o jefatura de servicios generales; dirección financiera o asistencia financiera; dirección o coordinación de proyectos de inversión; administración logística y de negocios internacionales; asesoría y consultoría empresarial; gerencia de su propia empresa.

Con el fin de cumplir el objetivo principal de este artículo, se dispone de una guía metodológica para definir el perfil profesional de programas académicos en IES, con la vinculación de la herramienta Ábaco de Régnier, para la obtención de conceptos y criterios que hagan prevalecer la opinión de todos los participantes, objetivamente y sin influencias, brindando resultados precisos (Mojica, 2006). Esta guía estandariza los pasos en el proceso de definición de perfiles profesionales de un programa académico de una IES. Conjuntamente, permite obtener información sobre actividades propias del egresado en su desempeño, necesidades de formación y problemas profesionales lo que converge en la determinación de competencias para un buen desempeño profesional.

\section{CONCLUSIONES}

De los resultados obtenidos, se pueden anunciar las siguientes conclusiones sobre la guía: 1) Permite su aplicación para definir el perfil profesional en cualquier IES; 2) Provee una solución innovadora acorde a las necesidades para cualquier programa académico; 3) Estandariza los parámetros para el diseño del perfil profesional; 4) Establece los lineamientos suficientes para realizar procesos de seguimiento y control; 5) Dispone información de apoyo de: Referentes teóricos, programas afines, documentos gubernamentales y de tendencias que ayudan a sustentar la pertinencia del futuro profesional; y 6) Consolida información de mapas de problemas y necesidades de formación en las competencias que establecen el perfil de egreso y garanticen su inserción laboral.

La guía conlleva un valor agregado importante: la sistematización de las fases en el proceso de definición del perfil profesional facilita disponer de la información aun cuando el proceso se suspenda. Si eso sucede podría reanudarse en cualquier fase y tiempo sin perder información, ahorrando costos y esfuerzos que representa principalmente la fase de recolección de información con los expertos. Finalmente, la sencillez con la que se describe la guía, contribuye a la comprensión y aplicación en cualquier programa académico; permitiendo además, el seguimiento de las etapas en la que se encuentra el proceso de definición del perfil profesional, y por ende disponer del control de procedimientos realizados.

\section{REFERENCIAS}

Alba, C., Un nuevo perfil profesional: enfoque prospectivo para el 2015, Ejército: de tierra español: $N^{\circ} 828$, 82-90 (2010)

Araya, W., Reflexiones e insumos sobre los Sistemas de Información Gerencial en la gestión de las instituciones de educación superior: Ventajas y desventajas. Gestión de la Educación: 5(2), 23-37 (2015) 
Arias, S. A.; Murillo, R. H.; Piñeros, M.; Bravo, M. M.; Hernández, G.; Pardo, C.; Quintero, A.; Rivera, D.; Sánchez, R.; Venegas, M.; Wiesner, C., Prioridades de Investigación para el Control del Cáncer en Colombia, Revista Colombiana de Cancerología 11(3):155-167 (2007)

ASCOLFA Asociación Colombiana de Facultades de Administración, Las competencias de los administradores en Colombia a la Luz del Proyecto Tuning América Latina. 2008-2010. (2010)

Bañuls, V.A. y J. L. Salmerón, Áreas clave para desarrollo económico y social: una visión desde la actividad prospectiva internacional, Problemas del Desarrollo. Revista Latinoamericana de Economía: 39(153) (2008)

Beneinote, P.; Esquetini, C.; González, J.; Marty, M; Siufi G. y R. Wagenaar, Reflexiones y perspectivas de la Educación Superior de América Latina, Informe final - Proyecto Tunin - América Latina 2004-2007, Universidad de Deusto, Bilbao (2007)

Borko, H., Information science: what is it? American documentation: 19(1), 3-5 (1968).Valero, G.M., Las competencias de los administradores en Colombia a la Luz del Proyecto Tuning América Latina, Revista puente científica: 5(1) (2011)

Canquiz, L.; Inciarte, A., Metodología para el Diseño de Perfiles basados en el Enfoque de Competencias, vol.15, núm. 29, enero-abril, (2009), Universidad Pedagógica Experimental Libertador, Caracas, Venezuela.

Cassingena, J., Vision Document, eFORESEE Malta ICT and Knowledge Futures Pilot (2003)

Castellanos, O.; Fonseca, S. L. y D.C. Ramírez, Retos de la ingeniería para el desarrollo tecnológico de la agroindustria. Revista de ingeniería: №33, 88-98 (2011)

Correa, J., Examinado el futuro: metodología propuesta para el estudio prospectivo y estratégico de la Universidad de Cundinamarca (2010-2019), Esquemas pedagógicos, (2010)

Escobar, M.E., Lineamientos para solicitud, otorgamiento y renovación de registro calificado. Programas de pregrado y posgrado (en la web: http://www.mineducacion.gov.co/1759/articles-338177_archivo_pdf.pdf, acceso: 2 de abril 2016), Ministerio de Educación Nacional (2013)

Godet, M., La caja de herramientas de la prospectiva estratégica, 4⿳a. Edición, Cuadernos № 5, Abril. (2007)

Godet, M., Prospectiva Estratégica: Problemas y Métodos. 2ª Edición, Cuadernos № 20, Enero. (2007)

Griffin-Sobel, J. P., The ENTREE model for integrating technologically rich learning strategies in a school of nursing. Clinical Simulation in Nursing: 5(2), e73-e78. (2009)

Hernández, E.; León, I.; Mesa, L. y E. González, Prospectiva Tecnológica en empresas de la industria alimentaria con apoyo del Análisis de Procesos. Centro Azúcar: 39(1), 58-67 (2012)

Huergo-Tobar, P. L. y O. Nicholls-Toro, Factores que inciden en la disminución en el ingreso de estudiantes en los programas de Administración de Empresas y Contaduría Pública de la Universidad Cooperativa de Colombia, sede Neiva. Estrategias: 12(22), 101-113 (2014)

Maringe, F., University and course choice: Implications for positioning, recruitment and marketing, International Journal of Educational Management: 20(6), 466-479 (2006)

Martelo, R.; Moncaris, L. y L. Velez, Plataforma web estudios de prospectiva Softprosp (2014)

Martelo, R.; Ponce, A. y F. Acuña, Guía Metodológica para el Diseño de un Plan Estratégico Informático en Instituciones de Educación Superior. Formación universitaria: 9(1), 91-98 (2016)

Mojica, F., El Ábaco de Régnier. En La Prospectiva, 21-33, Legis Editores, Bogotá, Colombia (1991)

Mojica, F., Concepto y aplicación de la Prospectiva Estratégica. Revista Med: 14(1), 122-131 (2006)

Morales, Y.; Fonseca, J. y M. García, En búsqueda de un perfil académico-profesional del personal docente de matemáticas. Unión: revista iberoamericana de educación matemática: № 38, 85-101 (2014)

Ortega, I.L.; Cruz, S.; Bermúdez, I.; Reyes, I.; Dupotey, N. y A. Ochoa, Perfil profesional del Licenciado en Farmacia de la Universidad de Oriente, Cuba, Revista Cubana de Farmacia: 45(1), 127-133 (2011) 
Pacheco, L. A., Perfil gerencial para las instituciones prestadoras de salud en Barranquilla. Pensamiento y gestión: № 25, 139-160 (2008)

Pineda, L., Prospectiva estratégica en la gestión del conocimiento: Una propuesta para los grupos de investigación colombianos. Investigación \& desarrollo: 21(1), 237-311 (2013)

Quiroga, D., Metodología para hacer prospectiva empresarial en la sociedad de la información y el conocimiento, Economía y Administración: 45(70), 25-44 (2008)

Sadosky, M., Historia de las TIC: principales movimientos y producciones (en la web: http://www.aportes.educ.ar/sitios/aportes/recurso/index?rec_id=107400\&nucleo=matematica_nucleo_tic, acceso: 4 de abril 2016), Educ.ar, El Portal Educativo del Estado Argentino (2000)

Sánchez, L.E.; Rosado, D.G.; Mellado, D.; Santos-Olmo, A. y E. Fernández-Medina, Proceso de Reificación de las Competencias Generales y Específicas para el Grado en Ingeniería Informática y Definición de un Plan de Métricas de Evaluación de dichas Competencias, XVII Jornadas de Enseñanza Universitaria de la Informática(JENUI11), 51-58 (2011)

Sierra, W.; Ortiz, A. N.; Alvarado, M. M.; Rangel, O. I., Agenda Prospectiva Estratégica de Ciencia y Tecnología, una Experiencia Significativa en la Planificación y Desarrollo Institucional: caso Universidad de La Guajira, Sotavento M.B.A. No. 22, 28-43 (2013)

Tobón, S.; Guzmán, C.E.; Hernández, J.S. y S. Cardona, Sociedad del Conocimiento: Estudio documental desde una perspectiva humanista y compleja. Revista Paradigma: 36(2), 19 (2015)

Tovar, M.C. y P. Sarmiento, El Diseño Curricular, una responsabilidad compartida. Colombia Médica: 42(4), 508-517 (2011)

UNESCO, Declaración Mundial sobre la Educación Superior en el Siglo XXI: Visión y Acción. Conferencia Mundial sobre la Educación (1998)

Velázquez, I.; Chequer, G.; Budan, P.; Sosa, M. y J. Reyes, Fundamentación epistemológica de la informática educativa como espacio interdisciplinar. Memorias del Congreso lberoamericano de Ciencia, Tecnología, Innovación y Educación, Buenos Aires, Argentina (2014)

Vidal, M. J. y A. B. Araña, Gestión de la información y el conocimiento, Revista Cubana de Educación Médica Superior: 26(3), 474-484 (2012) 